\title{
High $\beta$-Glucan Barley Improves Postprandial Hyperglycemia after Meal Loading in Patients with Type 2 Diabetes
}

\author{
Yukie Fuse ${ }^{1,2}$, Mariko Higa ${ }^{2 *}$, Asami Fujitani ${ }^{3}$, \\ Takamasa Ichijo ${ }^{2}$, Seiichiro $\mathbf{A o e}^{4}$ and Takahisa \\ Hirose $^{1}$ \\ ${ }^{1}$ Division of Diabetes, Metabolism, and Endocrinology, \\ Department of Medicine, Toho University Graduate \\ School of Medicine, Tokyo, Japan \\ ${ }^{2}$ Division of Diabetes and Endocrinology, Department \\ of Medicine, Saiseikai Yokohamashi Tobu Hospital, \\ Kanagawa, Japan \\ ${ }^{3}$ Nutrition Support Team, Saiseikai Yokohamashi Tobu \\ Hospital, Kanagawa, Japan \\ ${ }^{4}$ Department of Food Science, Faculty of Home \\ Economics, Otsuma Women's University, Tokyo, Japan \\ *Correspondling author: Mariko Higa, Division \\ of Diabetes and Endocrinology, Department of \\ Medicine, Saiseikai Yokohamashi Tobu Hospital, 3-6-1 \\ Shimosueyoshi, Tsurumi-ku, Yokohama-shi, Kanagawa \\ 230-0012, Japan
}

Received: February 09, 2021; Accepted: March 15, 2021; Published: March 22, 2021

\begin{abstract}
Objective: We investigated whether barley with a high $\beta$-glucan content (7.2 g per $100 \mathrm{~g}$ ) improves postprandial plasma glucose levels and fluctuations using a meal tolerance test and Continuous Glucose Monitoring (CGM) in patients with type 2 diabetes.

Methods: A meal tolerance test (500 kcal) was conducted using two types of test meals: one with white rice (WR diet) and one with white rice mixed 1:1 with high $\beta$-glucan-containing barley (BR diet). Both meals included the same side dish. Plasma glucose changes over 180 minutes were compared after ingestion of the test meals. In addition, participants wore a CGM device for the 2 study days, to measure the daily variations in glucose levels when the WR or BR diet as staple food was provided 3 times a day with the same side dish in both meals.
\end{abstract}

Results: Twenty-nine patients with type 2 diabetes (age $52.5 \pm 15.1$ years, BMI $27.5 \pm 4.7 \mathrm{~kg} / \mathrm{m}^{2}, \mathrm{HbA} 1 \mathrm{c} 8.1 \pm 1.8 \%$ ) were included in this study. The incremental area under the curve of plasma glucose levels after BR diet ingestion was significantly lower than that after WR diet ingestion (7934.0 \pm 4151.2 vs $9434.0 \pm 4701.2 \mathrm{mg} \cdot \mathrm{min} / \mathrm{dL}$ ). CGM showed a 24 -hour standard deviation of blood glucose after the BR diet was significantly lower than that after the WR diet.

Conclusion: These results suggest that postprandial plasma glucose elevation and fluctuation in patients with type 2 diabetes are suppressed by adding high- $\beta$-glucan barley to white rice in meals.

Keywords: Diet therapy; Beta glucan; Barley; Postprandial hyperglycemia; Type 2 diabetes

\section{Introduction}

The association between diabetes and a marked increase in the risk of coronary heart disease is well-established [1,2]. Although many factors are involved in the development of atherosclerosis and coronary heart disease, the two most important are hyperglycemia and insulin resistance [2,3]. There is growing evidence that the generation of oxidative stress and inflammation that occurs during postprandial hyperglycemia is an important mechanism for the initiation and progression of endothelial dysfunction in type 2 diabetes $[3,4]$. The postprandial increase in plasma glucose level is primarily governed by the amount of carbohydrate in the meal [57] and may vary depending on the quality of the carbohydrate [8]. Carbohydrate quality is assessed by the Glycemic Index (GI), which ranks carbohydrates based on the rate of glycemic response [8]. A recent study revealed that dietary fiber intake was inversely associated with all-cause mortality [9]. Brown rice and barley have a lower GI value and suppress postprandial elevation of plasma glucose level because they contain a higher amount of dietary fiber than White Rice (WR) [10-12]. Barley, in particular, contains a high amount of insoluble and soluble dietary fiber, and most of the soluble dietary fiber is $\beta$-glucan $[13,14]$, which may reduce the risk of cardiovascular diseases in part through its blood cholesterol-lowering action [15].

High intake of carbohydrate, including WR, causes an increase in postprandial plasma glucose level and is associated with an increased risk of cardiovascular disease, metabolic syndrome and diabetes $[16,17]$. Intake of high $\beta$-glucan barley leads to a reduction in visceral fat [18], and we previously showed that it suppressed postprandial plasma glucose elevation and oxidative stress, as well as reducing the mean blood glucose level over 24 hours, and blood glucose fluctuations, in volunteers with normal glucose tolerance [19].

Here, we conducted meal tolerance tests in patients with type 2 diabetes, using a test meal of WR mixed with two-rowed hull-less barley, which contains more $\beta$-glucan $(7.2 \mathrm{~g}$ per $100 \mathrm{~g})$ than common barley [20], to examine whether high $\beta$-glucan barley improves postprandial plasma glucose levels. In addition, Continuous Glucose Monitoring (CGM) system was used to assess blood glucose fluctuations of the day.

\section{Materials and Methods}

\section{Study participants}

Participants were recruited among patients admitted to Saiseikai
Austin J Nutr Metab - Volume 8 Issue 2 - 2021

Submit your Manuscript | www.austinpublishinggroup.com

Higa et al. (C) All rights are reserved
Citation: Fuse Y, Higa M, Fujitani A, Ichijo T, Aoe S and Hirose T. High $\beta$-Glucan Barley Improves Postprandial Hyperglycemia after Meal Loading in Patients with Type 2 Diabetes. Austin J Nutr Metab. 2021; 8(2): 1104. 
Yokohamashi Tobu Hospital for diabetic control between January 2015 and August 2015. Twenty-nine patients with type 2 diabetes (15 men and 14 women) were included. The inclusion criteria were as follows: 1) age $<75$ years; 2) fasting plasma glucose levels $\leq 140 \mathrm{mg} /$ $\mathrm{dL}$; 3) daily plasma glucose fluctuations $<100 \mathrm{mg} / \mathrm{dL}$ as shown in Table 1. Patients diagnosed with severe renal dysfunction (estimated glomerular filtration rate $<50 \mathrm{~mL} / \mathrm{min} / 1.73 \mathrm{~m}^{2}$ ) and/or serious cardiovascular diseases were excluded. Patients were also required to not have eaten food containing barley for 1 week prior to the study. This was confirmed using a dietary survey. Study participants had a mean age of $57.2 \pm 15.1$ years. Mean body mass index, fasting plasma glucose and HbA1c levels were $27.5 \pm 4.7 \mathrm{~kg} / \mathrm{m}^{2}, 119.3 \pm 16.1$ $\mathrm{mg} / \mathrm{dL}$, and $8.1 \pm 1.8 \%$, respectively. Three patients were treating their diabetes with diet alone, 15 patients were receiving insulin with oral hypoglycemic agents, and 11 patients were receiving oral hypoglycemic agents alone. Of the 29 patients treated with insulin or oral hypoglycemic agents, 14 were receiving a Dipeptidyl Peptidase- 4 (DPP-4) inhibitor. All treatments, including oral antidiabetic agents and doses of insulin, remained unchanged throughout the study period.

The study was conducted in accordance with the Declaration of Helsinki and its subsequent amendments. All procedures were approved by the Saiseikai Yokohamashi Tobu Hospital ethics committee (Ethics committee file number 2013030), and written informed consent was obtained from all patients.

\section{Meal tolerance test}

The meal tolerance test was performed in the morning after overnight fast, with no food or drink after 21:00 the evening before the test. Early in the morning, on an empty stomach, the patients consumed two types of diet adjusted to $500 \mathrm{kcal}$ in about 15 minutes, and oral hypoglycemic drugs and insulin injections were administered in normal doses, remained unchanged throughout the study period. The meal tolerance test was performed on 2 consecutive days in randomized crossover design. Two different diets were prepared: one consisted of WR with a side dish (WR diet), and the other was WR mixed 1:1 with $\beta$-glucan rich barley (BR) with the same side dish as in the WR diet (BR diet). The patients were randomized into two groups. One had the WR diet on the first day and the BR diet the next day, and the other had the BR diet on the first day and the WR diet the next day. For lunch and dinner after the breakfast tolerance test, the main dish was $\mathrm{WR}$ for the WR diet and $\mathrm{BR}$ for the $\mathrm{BR}$ diet.

The specific barley used in this study was "Kirarimochi". Kirarimochi was registered as a new breed of two-rowed hull-less barley in 2009 and has a high $\beta$-glucan content $(7.2 \mathrm{~g}$ per $100 \mathrm{~g}$ ) [20], about 1.5 times higher than that of normal covered barley ( $4.7 \mathrm{~g}$ per $100 \mathrm{~g}$ ). This barley type was developed by the National Agriculture and Food Research Organization in Japan, and is not a genetically modified crop. The high $\beta$-glucan barley was pearled to remove the bran (pearled to $60 \%$ yield) and the remaining $60 \%$ was rice-shaped barley, which was used in the meal [20]. The BR diet consisted of 50\% Kirarimochi and 50\% WR.

The WR diet comprised $504 \mathrm{kcal}, 80 \mathrm{~g}$ carbohydrate (glucose 76.9 $\mathrm{g}$, dietary fiber $3.5 \mathrm{~g}$ ), $18.7 \mathrm{~g}$ protein, and $11.4 \mathrm{~g}$ lipid. In comparison, the BR diet was $479 \mathrm{kcal}$, comprising $75 \mathrm{~g}$ carbohydrate (glucose 68.8 $\mathrm{g}$, dietary fiber $6.3 \mathrm{~g}$ ), $18.9 \mathrm{~g}$ protein and $11.6 \mathrm{~g}$ lipid. The amount of staple food was adjusted to $150 \mathrm{~g}$ for both WR and BR diets.

Plasma glucose was measured before the test meal and 30, 60, 120, and 180 minutes after. The Incremental Area Under the Curve (IAUC) of plasma glucose levels was calculated using the trapezoidal method [21] from values up to 180 minutes after ingestion, with the value at fasting state as baseline. Plasma triglyceride and free fatty acid (FFA) levels were measured before the meal and 60 and 180 minutes after. Plasma triglyceride and FFA levels were measured using enzymatic methods (BML, Tokyo, Japan). Serum high-sensitivity CRP (h-CRP) and urinary 8-hydroxydeoxyguanosine (8-OHdG) levels were also measured. Urinary 8-OHdG was corrected with urinary creatinine. Enzyme-linked immunosorbent assay methods (SRL, Tokyo, Japan) were used for the measurements of h-CRP and urinary 8-OHdG.

\section{Continuous Glucose Monitoring (CGM) system}

The 29 subjects who underwent the meal tolerance test continued to participate in the CGM study. A CGM system was fitted to examine the daily fluctuation in blood glucose after meals with WR or BR as the main dish. The test was conducted in a randomized crossover design and the patients were divided into two groups: one had the WR diet as the main dish for breakfast, lunch, and dinner on the first day and the BR diet at equivalent times the next day, and the other had the BR diet as the main dish for breakfast, lunch, and dinner on the first day and the WR diet at equivalent times the next day. The side dishes were the same in both groups. Oral hypoglycemic drugs and insulin injections were administered in usual doses, remained unchanged throughout the study period, and activity was adjusted to remain the same as far as possible over the 2 days.

The daily calorie intake was adjusted to $1800 \mathrm{kcal}$ for men and $1600 \mathrm{kcal}$ for women. The diets were prepared with identicallooking content and number of side dishes. For $1600 \mathrm{kcal}$ per day, the WR diet contained $1677 \mathrm{kcal}$ and $64.5 \mathrm{~g}$ protein, $47.1 \mathrm{~g}$ lipid and $241 \mathrm{~g}$ carbohydrate (glucose $232.5 \mathrm{~g}$, dietary fiber $7.2 \mathrm{~g}$ ), and the BR diet contained $1602 \mathrm{kcal}, 65.1 \mathrm{~g}$ protein, $47.7 \mathrm{~g}$ lipid and $226 \mathrm{~g}$ carbohydrate (glucose 209 g, dietary fiber 16.9 g). For $1800 \mathrm{kcal}$ per day, protein and lipid content were identical to the $1600 \mathrm{kcal}$ diet, but the WR diet contained $291 \mathrm{~g}$ carbohydrate (glucose $280 \mathrm{~g}$, dietary fiber $8.6 \mathrm{~g}$ ), and the BR diet contained $276 \mathrm{~g}$ carbohydrate (glucose $250 \mathrm{~g}$, dietary fiber $20.3 \mathrm{~g}$ ).

CGM was carried out using the $\mathrm{iPro}^{\circ} 2$ (Medtronic, MiniMed Northridge, CA, USA), which was placed on each participant's abdomen before lunch on the day before the test. The following CGM variables were calculated: daily mean blood glucose levels (24-hour MBG), 24-hour standard deviation of blood glucose (24-hour SDBG), and Mean Amplitude of Glucose Excursion (MAGE) [22]. MAGE is an index of glycemic variability calculated from the value larger than one standard deviation (SD) from the mean glycemic values \pm SD. The other indexes of glycemic variability were calculated as follows; coefficient of variance $(\% \mathrm{CV})=\mathrm{SD} / \mathrm{MBG} \times 100$, and $\mathrm{J}$-index $=0.001$ $\times(\mathrm{MBG}+\mathrm{SD})^{2}[22]$.

\section{Statistical analysis}

Results are expressed as the mean $\pm \mathrm{SD}$. Temporal changes in plasma glucose, triglyceride, FFA, h-CRP and urinary 8-OHdG levels from the fasting state up to 180 minutes after ingestion were evaluated by paired Student's $t$-tests. When the variables did not 
Table 1: Clinical characteristics of subjects.

\begin{tabular}{|l|c|c|}
\hline \multicolumn{2}{|c|}{ Type 2 diabetic patients $\mathbf{n = 2 9}$} \\
\hline Age (years) & $57.2 \pm 15.1$ & \\
\hline Gender (male/female) & $15 / 14$ & \\
\hline Body mass index $\left(\mathrm{kg} / \mathrm{m}^{2}\right)$ & $27.5 \pm 4.7$ & \\
\hline HbA1c $(\%)$ & $8.1 \pm 1.8$ & \\
\hline Fasting plasma glucose $(\mathrm{mg} / \mathrm{dL})$ & $119.3 \pm 16.1$ & \\
\hline Duration of diabetes (years) & $6.3 \pm 5.9$ & $\mathrm{n}=3$ \\
\hline & Diet alone & $\mathrm{n}=11$ \\
\hline Diabetic treatment & OH agents & 15 \\
& Insulin treatment & $14 / 15$ \\
\hline
\end{tabular}

$\mathrm{OH}$ agents: Oral Hypoglycemic agents; DPP-4 inhibitor: Dipeptidyl Peptidase-4 inhibitor.

Values are mean \pm standard deviations

exhibit a normal distribution, the Wilcoxon rank-sum test was used to compare the two groups. Differences between WR and BR diet were evaluated using unpaired Student's $t$-tests, and Welch's $t$-test was used for analysis if equal variances were not assumed. The MannWhitney $\mathrm{U}$ test was used to compare the IAUC of plasma glucose levels between the WR and BR diets. $\mathrm{p}<0.05$ in a two-sided test was considered significant. SAS JMP version 11 (SAS Institute Inc., Cary, NC, USA) was used for all statistical analyses.

\section{Results}

\section{Meal tolerance test}

Plasma glucose level before ingestion of the WR diet was 114.7 $\pm 16.8 \mathrm{mg} / \mathrm{dL}$, and this increased significantly to a peak of $173.3 \pm$ $38.9 \mathrm{mg} / \mathrm{dL}$ at 60 minutes after ingestion $(\mathrm{p}<0.0001)$, and returned to $150.7 \pm 33.1 \mathrm{mg} / \mathrm{dL}$ at 180 minutes after ingestion. Basal plasma glucose level in the BR diet was $114.6 \pm 16.6 \mathrm{mg} / \mathrm{dL}$, the peak value was $165.2 \pm 27.2 \mathrm{mg} / \mathrm{dL}$ at 60 minutes after ingestion, which was significantly higher than the basal value $(\mathrm{p}<0.0001)$, and it returned to $140.4 \pm 26.5 \mathrm{mg} / \mathrm{dL}$ at 180 minutes. Plasma glucose levels at 180 minutes in the BR diet were significantly lower than those in the WR $\operatorname{diet}(\mathrm{p}<0.05)$, but there was no significant difference between the WR and $\mathrm{BR}$ diets in plasma glucose levels at 60 minutes. As indicated in Table 2, the IAUC of plasma glucose levels over 180 minutes after ingestion of the BR diet was significantly lower $(\mathrm{p}<0.01)$ than that after ingestion of the WR diet. Plasma triglyceride levels were significantly higher 180 minutes after ingestion of the WR or BR diets than at baseline $(\mathrm{p}<0.001)$, and its level after ingestion of the BR diet showed significant high compared to those after the WR diet $(\mathrm{p}<0.001)$. FFA levels 180 minutes after ingestion of the test meals were significantly lower than those before ingestion levels, with no significant difference between the two groups as shown Table 3. No significant changes were seen in urinary $8-\mathrm{OHdG}$ and h-CRP before and after ingestion of WR diet or BR diet.

\section{Continuous Glucose Monitoring (CGM) system}

Table 4 depicts the results of the parameters of glycemic variability.

Table 2: The Incremental Area Under the Curve (IAUC) of blood glucose levels over 180 minutes after the consumption of test meals with White Rice alone (WR diet) or white rice mixed 1:1 with $\beta$-glucan rich barley (BR diet).

\begin{tabular}{|c|c|c|c|}
\hline & WR diet & BR diet & P value \\
\hline IAUC mg/min/dL & $9434.0 \pm 4701.2$ & $7934.0 \pm 4151.2$ & $<0.01$ \\
\hline
\end{tabular}

Values are mean \pm standard deviations.
There was no significant difference in 24-hour MBG level between the WR and BR diets. The 24-hour SDBG and \%CV were significantly lower for the BR diet than the WR diet $(\mathrm{p}<0.05)$; however, no significant difference in MAGE and J-index between the WR and BR diets was observed. In addition, the daily fluctuation in blood glucose levels were compared across diabetes treatment modalities. In the 14 patients who were taking a DPP-4 inhibitor, MAGE for WR diet was significantly lower than in the 15 patients who were not (43.7 \pm 17.9 $\mathrm{mg} / \mathrm{dl} v s 60.5 \pm 26.4 \mathrm{mg} / \mathrm{dl} ; \mathrm{p}<0.05$ ), and also MAGE for BR diet in the 14patients taking a DPP-4 inhibitor was significantly lower than in the 15 patients who were not $(40.7 \pm 20.9 \mathrm{mg} / \mathrm{dl} v s 61.0 \pm 24.8$ $\mathrm{mg} / \mathrm{dl} ; \mathrm{p}<0.05)$. However, the differences between WR diet and BR diet in two groups were not significant. No significant differences in parameters of glycemic variability were seen between patients with and without insulin treatment.

\section{Discussion}

We previously reported that postprandial glucose elevation and glucose fluctuations were suppressed by ingestion of a mix of high $\beta$-glucan barley and WR in volunteers with normal glucose tolerance [19]. Similarly, the present study showed that high $\beta$-glucan barley also ameliorated postprandial hyperglycemia and glucose level fluctuations in patients with type 2 diabetes. Therefore, high $\beta$-glucan barley might decrease postprandial hyperglycemia not only in healthy people but also in people with type 2 diabetes. At least 4 $\mathrm{g}$ of $\beta$-glucan and $30-80 \mathrm{~g}$ available carbohydrate can significantly reduce postprandial blood glucose [23]. Conventionally, $\beta$-glucan content in barley is reported to be $4.7 \mathrm{~g}$ per $100 \mathrm{~g}$. In contrast, the Kirarimochi barley used in this study has a content of $\beta$-glucan as high as $7.2 \mathrm{~g}$ per $100 \mathrm{~g}$ [20]. In this study, the composition of the BR diet was almost $4 \mathrm{~g}$ of $\beta$-glucan and $54 \mathrm{~g}$ available carbohydrate, fulfilling Tosh's recommendation [23]. In healthy people, preloading with $\beta$-glucan mixed in water before a meal reduced the glucose AUC

Table 3: Changes in plasma lipid levels after consumption of test meals with white rice alone (WR diet) or white rice mixed 1:1 with $\beta$-glucan rich barley (BR diet).

\begin{tabular}{|c|c|c|c|c|}
\hline \multicolumn{2}{|c|}{} & $0 \mathrm{~min}$ & $60 \mathrm{~min}$ & $180 \mathrm{~min}$ \\
\hline Triglyceride $(\mathrm{mg} / \mathrm{dL})$ & WR diet & $118.4 \pm 47.3$ & $121.1 \pm 46.6$ & $138.3 \pm 52.8^{*}$ \\
\cline { 2 - 5 } & BR diet & $125.8 \pm 50.5$ & $128.4 \pm 47.4$ & $155.9 \pm 58.4$ \\
\hline Free fatty acids $(\mathrm{mEq} / \mathrm{L})$ & WR diet & $0.52 \pm 0.19$ & $0.41 \pm 0.16$ & $0.27 \pm 0.14$ \\
\hline & BR diet & $0.51 \pm 0.17$ & $0.41 \pm 0.19$ & $0.28 \pm 0.14$ \\
\hline
\end{tabular}

: $p<0.001$ were obtained WR diet vs BR diet using unpaired student's $t$ test. Values are mean \pm standard deviations.

Table 4: The daily mean blood glucose and daily variation of blood glucose levels in Continuous Glucose Monitoring (CGM) system after consumption of White Rice alone (WR diet) or white rice mixed 1:1 with $\beta$-glucan rich barley (BR diet).

\begin{tabular}{|c|c|c|c|}
\hline & WR diet & BR diet & P value \\
\hline $24-h M B G ~ m g / d l$ & $133.6 \pm 18.1$ & $134.0 \pm 16.7$ & NS \\
\hline MAGE mg/dl & $52.4 \pm 23.9$ & $51.2 \pm 24.8$ & NS \\
\hline $24-h S D B G ~ m g / d l$ & $25.2 \pm 10.2$ & $22.2 \pm 10.7$ & $<0.05$ \\
\hline$\% C V$ & $18.9 \pm 7.5$ & $16.5 \pm 7.9$ & $<0.05$ \\
\hline J-index & $25.8 \pm 7.5$ & $24.9 \pm 7.1$ & NS \\
\hline
\end{tabular}

24-hMBG: 24-hour daily mean blood glucose, MAGE: mean amplitude of glucose excursion, 24-hSDBG: 24-hour standard deviation of blood glucose, \%CV: coefficient of variance.

Values are mean \pm standard deviations. 
and energy intake $[24,25]$, whereas in people with type 1 diabetes, $\beta$-glucan tablets did not improve glycemic control or glucose variability [26]. Insoluble dietary fiber improves bowel movement by increasing stool volume [27], whereas soluble dietary fiber, which is mixed with food inside the intestine, suppresses the acute elevation of glucose and serum cholesterol levels due to its slow movement within the intestine $[28,29]$. In addition, the high viscosity of soluble dietary fibers, especially $\beta$-glucan, results in them strongly delaying the digestion and absorption of nutrients [28]. Beta glucan is likely to be involved in complex processes and interactions with the food matrix [30]. The present findings show that a mix of high $\beta$-glucan barley and WR is effective in managing postprandial hyperglycemia. The American Diabetes Association recommends eating a moderate amounts of fiber (total, $24 \mathrm{~g} ; 8 \mathrm{~g}$ soluble fiber and $16 \mathrm{~g}$ insoluble fiber) [31]. In this study, $1800 \mathrm{kcal}$ of BR diet contained $20.3 \mathrm{~g}$ fiber for a day, approaching this recommendation, in contrast to the WR diet, which contained $8.6 \mathrm{~g}$.

Consumption of glutinous brown rice improved postprandial glucose excursion and insulin hypersecretion [12]. Glutinous brown rice contains gamma-oryzanol, which may improve metabolic parameters [12,32]. We previously reported that high $\beta$-glucan barley suppressed an increase in postprandial insulin levels, possibly by delaying digestion, absorption, and gastric emptying [33]. Meals that include vegetables and dietary fiber increase glucagon-like peptide 1 (GLP-1) more than meals of WR alone, and suppress hyperglycemia and excessive secretion of endogenous insulin after meals [34]. A previous study reported significant differences in the effects of glutinous brown rice between patients treated with and without DPP-4 inhibitors [12]. This may be explained by glutinous brown rice stimulating GLP-1 secretion as a result of increased production of short-chain fatty acids from dietary fiber by gut microbial flora [34,35]. In the present study, patients receiving a DPP-4 inhibitor showed smaller postprandial glucose fluctuations than those not receiving a DPP-4 inhibitor, regardless of whether they ate the WR or BR diet, which might association with a augmentation of plasma GLP-1 concentrations and slowing of gastric emptying, as suggested previously $[12,36]$. Consequently, it is difficult to say whether high $\beta$-glucan barley is effective for patients taking a DP- 4 inhibitor.

Soluble dietary fiber lowers serum cholesterol and triglycerides and improves intrahepatic lipid accumulation [37]. We observed no differences in serum triglyceride or FFA levels between the BR and WR diets. Longer-term observation studies are needed to clarify the effect of $\beta$-glucan barley on lipid metabolism. Mixing Kirarimochi barley with WR improves postprandial blood glucose levels and may prevent diabetes and metabolic syndrome $[18,38]$, as well as preventing cardiovascular disease by decreasing postprandial glucose fluctuations, insulin hypersecretion, and antioxidant status [39]. However, in this study the marker of oxidative stress and inflammation showed no changes during postprandial hyperglycemia. A longterm study is necessary to clarify whether $\beta$-glucan barley improves oxidative stress or inflammation after ingestion of the meal. Normal covered barley generally has a $\beta$-glucan content of about $4.7 \%$, whereas that of Kirarimochi is about 1.5-times higher. Further investigation is needed to clarify whether common barleys also reduce postprandial hyperglycemia and glucose fluctuation. Our study has limitation. First, the sample size was small and study duration was short. Second, there was no wash out period between WR diet and BR diet.

In conclusion, in the meal tolerance test and CGM study, IAUC for glucose over 180 minutes and glucose level fluctuation after ingestion were significantly decreased by the consumption of WR mixed 1:1 with high $\beta$-glucan barley in patients with type 2 diabetes. Lowering the GI of WR by adding $\beta$-glucan appears to suppress postprandial hyperglycemia. This might contribute to preventing obesity and suppressing the onset of vascular disease in Asian patients with diabetes who use WR as a staple food. Nonetheless, long-term research is still needed to clarify whether high $\beta$-glucan barley improves postprandial blood glucose levels.

\section{Acknowledgment}

The study was supported by a Research Project on Development of Agricultural Products and Food with Health-promoting Benefits awarded by National Agriculture and Food Research Organization (NARO), Japan. Authors thanks all those who participated in this study.

\section{Author Contributions}

Study concept and design: SA, AF and $\mathrm{MH}$; acquisition of data: YF, and MF; analysis and interpretation of data: TI, YF and MH; drafting of the manuscript: YF, MH and TH; critical revision of the manuscript: $\mathrm{MH}$ and $\mathrm{TH}$; statistical analysis: $\mathrm{TI}$; obtained funding: SA; administrative, technical, or material support: SA and AF; and study supervision: all authors.

\section{References}

1. Haffner SM, Lehto S, Ronnemaa T, Pyorala K, Laakso M. Mortality from Coronary Heart Disease in Subjects with Type 2 Diabetes and in Nondiabetic Subjects with and without Prior Myocardial Infarction. N Engl J Med. 1998; 339: 229-234.

2. Farjo PD, Barghouthi N, Chima N, Desai A, Fang W, Giordano J, et al. Use of the Burden of Diabetes Mellitus Score for Cardiovascular Disease Risk Assessment. Am J Cardiol. 2020; 125: 1829-1835.

3. Katakami N. Mechanism of Development of Atherosclerosis and Cardiovascular Disease in Diabetes Mellitus. J Atheroscler Thromb. 2018; 25: 27-39.

4. Ceriello A, Esposito K. Pieoni L, Ihnat MA, Thorpe JE, Testa R, et al. Oscillating Glucose Is More Deleterious to Endothelial Function and Oxidative Stress Than Mean Glucose in Normal and Type 2 Diabetic Patients. Diabetes. 2008; 57: 1349-1354.

5. Silva FM, Kramer CK, Crispim D, Azevedo MJ. A High-Glycemic Index, Low-Fiber Breakfast Affects the Postprandial Plasma Glucose, Insulin, and Ghrelin Responses of Patients with Type 2 Diabetes in a Randomized Clinical Trial. J Nutr. 2015; 145: 736-741.

6. Soong YY, Quek RY, Henry CJ. Glycemic Potency of Muffins Made with Wheat, Rice, Corn, Oat and Barley Flours: a comparative study between in vivo and in vitro. Eur J Nutr. 2015; 54: 1281-1285.

7. Truswell AS. Glycaemic Index of Foods. Eur J Clin Nutr. 1992; 46: S91-S101.

8. Brouns F, Bjorck I, Frayn KN, Gibbs AL, Lang V, Slama G, et al. Glycaemic Index Methodology. Nutr Res Rev. 2005; 18: 145-171.

9. Katagiri R, Goto A, Sawada N, Yamaji T, Iwasaki M, Noda M, et al. Dietary Fiber Intake and Total and Cause-Specific Mortality: the Japan public health center-based prospective study. Am J Clin Nutr. 2020; 111: 1027-1035.

10. Bui TN, Le TH, Nguyen DH, Tran QB, Nguyen TL, Le DT, et al. Pre-germinated Brown Rice Reduced Both Blood Glucose Concentration and Body Weight in Vietnamese Women with Impaired Glucose Tolerance. J Nutr Sci Vitaminol. 2014; 60: 183-187. 
11. Candido FG, Ton WTS, Alfenas RCG. Addition of Dietary Fiber Sources to Shakes Reduces Postprandial Glycemia and Alters Food Intake. Nutr Hosp. 2015; 31: 299-306.

12. Nakayama T, Nagai $\mathrm{Y}$, Uehara $\mathrm{Y}$, Nakamura $\mathrm{Y}$, Ishii $\mathrm{S}$, Tanaka $\mathrm{Y}$. Eating Glutinous Brown Rice Twice a Day for 8 Weeks Improves Glycemic Contro in Japanese Patients with Diabetes Mellitus. Nutr \& Diabetes. 2017; 7: e273.

13. Grundy MML, Quint J, Rieder A, Balance S, Dreiss CA, Cross KL, et al. The Impact of Oat Structure and $\beta$-glucan on in vitro Lipid Digestion. J Funct Foods. 2017; 38: 378-388.

14. Oda T, Aoe S, Sanada H, Ayano Y. Effects of Soluble and Insoluble Fibe Preparations Isolated from Oat, Barley, and Wheat on Liver Cholestero Accumulation in Cholesterol-fed Rats. J Nutr Sci Vitaminol (Tokyo). 1993 39: 73-79.

15. EFSA Panel on Dietetic Products, Nutrition and Allergies (NDA). Scientific Opinion on the Substantiation of a Health Claim Related to Barley Betaglucans and Lowering of Blood Cholesterol and Reduced Risk of (coronary) Heart Disease Pursuant to Article 14 of Regulation (EC) NO 1924/2006. EFSA J. 2011; 9: 2471.

16. Ley SH, Hamdy O, Mohan V, Hu F. Prevention and Management of Type 2 Diabetes: Dietary Components and Nutritional Strategies. Lancet. 2014; 383: 1999-2007.

17. Sakurai M, Nakamura K, Miura K, Takamura T, Yoshita K, Nagasawa S, et al. Dietary Carbohydrate Intake, Presence of Obesity and the Incident Risk of Type 2 Diabetes in Japanese Men. J Diabetes Investig. 2016; 7: 343-351.

18. Aoe S, Ichinose $Y$, Kohyama N, Komae K, Takahashi A, Abe D, et al. Effects of High $\beta$-Glucan Barley on Visceral Fat Obesity in Japanese Individuals: a randomized, double-blind study. Nutrition 2017; 42: 1-6.

19. Higa M, Fuse $Y$, Miyashita N, Fujitani A, Yamashita K, Ichijo T, et al. Effect of High $\beta$-Glucan Barley on Postprandial Blood Glucose Levels in Subjects with Normal Glucose Tolerance: assessment by meal tolerance test and continuous glucose monitoring system. Clin Nutr Res. 2019; 8: 55-63.

20. Yanagisawa T, Nagamine T, Takahashi A, Takayama T, Doi Y, Matsunaka H, et al. Breeding of Kirari-mochi: a new two-rowed waxy hull-less barley cultivar with superior quality characteristics. Breed Sci. 2011; 61: 307-310.

21. Sowunmi A, Gbotosho GO, Happi CT, Folarin O, Okuboyejo T, Michael O, et al. Use of Area Under the Curve to Evaluate the Effects of Antimalaria Drugs on Malaria Associated Anemia After Treatment. Am J Ther. 2011; 18 : 190-197.

22. Shivaprasad C, Aiswarya Y, Kejal S, Sridevi A, Anupam B, Ramdas B, et al. Comparison of CGM-derived Measures of Glycemic Variability Between Pancreatogenic Diabetes and Type 2 Diabetes Mellitus. J Diabetes Sci Technor. 2019; 15: 134-140.

23. Tosh SM. Review of Human Studies Investigating the Post-prandial Bloodglucose Lowering Ability of Oat and Barley Food Products. Eur J Clin Nutr. 2013; $67:$ 310-317

24. Steinert RE, Raederstorff D, Wolever TM. Effect of Consuming Oat Bran Mixed in Water Before a Meal on Glycemic Responses in Healthy Humans-A pilot study-Nutrients. 2016; 8: 524.

25. Aoe S, Ikenaga T, Noguchi H, Kohashi C, Kakumoto K, Kohda N. Effect of Cooked White Rice with High $\beta$-glucan Barley on Appetite and Energy Intake in Healthy Japanese Subjects: a randomized controlled trial. Plant Foods Hum Nutr. 2014; 69: 325-330.
26. Frid A, Tura A, Pacini G, Ridderstrale M. Effect of Oral Pre-meal Administration of Betaglucans on Glycaemic Control and Variability in Subjects with Type 1 Diabetes. Nutrients. 2017; 9: 1004

27. Saito T, Hayakawa T, Nakamura K, Takita T, Suzuki K, Innami S. Fecal Output, Gastrointestinal Transit Time, Frequency of Evacuation and Apparent Excretion Rate of Dietary Fiber in Young Men Given Diets Containing Different Levels of Dietary Fiber. J Nutr Sci Vitaminol (Tokyo). 1991; 37: 493-508.

28. Wang $Q$, Ellis PR. Oat $\beta$-glucan: physico-chemical characteristics in relation to its blood-glucose and cholesterol-lowering properties. Br J Nutr. 2014; 112 S4-13.

29. Yu K, Ke MY, Li WH, Zhang SQ, Fang XC. The Impact of Soluble Dietary Fibre on Gastric Emptying, Postprandial Blood Glucose and Insulin in Patients with Type 2 Diabetes. Asia Pac J Clin Nutr. 2014; 23: 210-218.

30. Grundy MM, Quint J, Rieder A, Ballance S, Dreiss CA, Cross KL, et al. The Impact of Oat Structure and $\beta$-glucan on in Vitro Lipid Digestion. J Funct Foods. 2017; 38: 378-388.

31. Chandalia M, Garg A, Lutjohann D, Bergmann K, Grundy SM, Brinkley LJ. Beneficial Effects of High Dietary Fiber Intake in Patients with Type 2 Diabetes Mellitus. N Engl J Med. 2000; 342: 1392-1398.

32. Kozuka C, Yabiku K, Takayama C, Matsushita M, Shimabukuro M. Natura Food Science Based Novel Approach Toward Prevention and Treatment of Obesity and Type 2 Diabetes: recent studies on brown rice and y-oryzanol. Obes Res Clin Pract. 2013; 7: e165-e172.

33. Fuse $Y$, Higa M, Miyashita N, Fujitani A, Yamashita K, Ichijo T, et al. Effect of High $\beta$-Glucan Barley on Postprandial Blood Glucose and Insulin Levels in Type 2 Diabetic Patients. Clin Nutr Res. 2020; 9: 43-51.

34. Kameyama N, Maruyama C, Matsui S, Araki R, Yamada Y, Maruyama T. Effects of Consumption of Main and Side Dishes with White Rice on Postprandial Glucose, Insulin, Glucose-dependent Insulinotropic Polypeptide and Glucagon-like Peptide-1 Responses in Healthy Japanese Men. Br J Nutr. 2014; 111: 1632-1640.

35. Zou J, Chassaing B, Singh V, Pellizzon M, Ricci M, Fythe MD, et al. Fibermediated Nourishment of Gut microbiota Protects Against Diet-induced Obesity by Restoring IL-22-mediated Colonic Health. Cell Host \& Microbe. 2018; 23: 41-53.e4.

36. Drucker DJ, Nauck MA. The Incretin System: glucagon-like peptide-1 receptor agonists and dipeptidyl peptidase- 4 inhibitors in type 2 diabetes. Lancet. 2006; 368: 1696-1705.

37. Brockman DA, Chen X, Gallaher DD. Consumption of a High $\beta$-glucan Barley Flour Improves Glucose Control and Fatty Liver and Increases Muscle Acylcarnitines in the Zucker Diabetic Fatty Rat. Eur J Nutr. 2013; 52: 17431753.

38. Galisteo M, Duarte J, Zarzuelo A. Effects of Dietary Fibers on Disturbances Clustered in the Metabolic Syndrome. J Nutr Biochem. 2008; 19: 71-84.

39. Gao R, Wang Y, Wu Z, Ming J, Zhao G. Interaction of Barley $\beta$-glucan and Tea Polyphenols on Glucose Metabolism in Streptozotocin-induced Diabetic Rats. J Food Sci. 2012; 77: H128-H134. 